Doug Geisler, Eva K. Grebel, and Dante Minniti, eds.

\title{
Dynamical Evolution of Rich Star Clusters in the LMC
}

\author{
Mark I. Wilkinson, Gerard F. Gilmore \\ Institute of Astronomy, Madingley Road, Cambridge CB3 OHA, UK \\ Jarrod Hurley \\ American Museum of Natural History, New York, USA
}

\begin{abstract}
We combine the results of a large HST study of rich LMC clusters with state of the art $N$-body simulations. We summarise recent highlights from our project: the estimation of age spreads in young LMC clusters, the measurement of the binary fraction of NGC1818, and the determination of deep cluster luminosity functions (LF). We also present preliminary results from an investigation of the core radius versus age relation evident among LMC clusters.
\end{abstract}

\section{Introduction - HST Project GO-7307}

The rich star clusters of the LMC provide a unique test-bed for ideas concerning the formation and evolution of star clusters. We find LMC clusters with masses comparable to those of Galactic globular clusters but with ages ranging from a few dynamical crossing times up to $10^{10}$ years. The youngest clusters are not yet fully dynamically relaxed and may therefore provide insight into the initial conditions of cluster formation. The main sequences (MS) of these clusters contain a great deal of information about the initial mass function (IMF) of stars because a wide range of stellar masses can be observed on the MS. The relative proximity of the clusters together with the lack of foreground obscuration aids the interpretation of deep colour magnitude diagrams (CMDs).

HST project GO-7307 (Beaulieu et al. 1999) was conceived to confront the results of detailed HST observations of LMC clusters with realistic $N$-body simulations in order to build a coherent picture of cluster evolution. In particular, we intend to use all the information about the IMF, binary fraction and degree of primordial mass segregation obtained from young LMC clusters to constrain the initial conditions for our $N$-body models. We have obtained deep (V $\sim 26$ ) images of eight rich LMC clusters with the HST WFPC2 (V, I), NICMOS (J, $\mathrm{H})$ and STIS $(\mathrm{R}+\mathrm{I})$ instruments. The target clusters are paired in age with two young clusters, four of intermediate ages and two old clusters, in order to allow comparison of cluster properties at a range of evolutionary epochs. All the clusters are currently located at similar projected distances from the LMC and the clusters were chosen such that each pair exhibits a range in at least one observable, such as core radius or metallicity. 


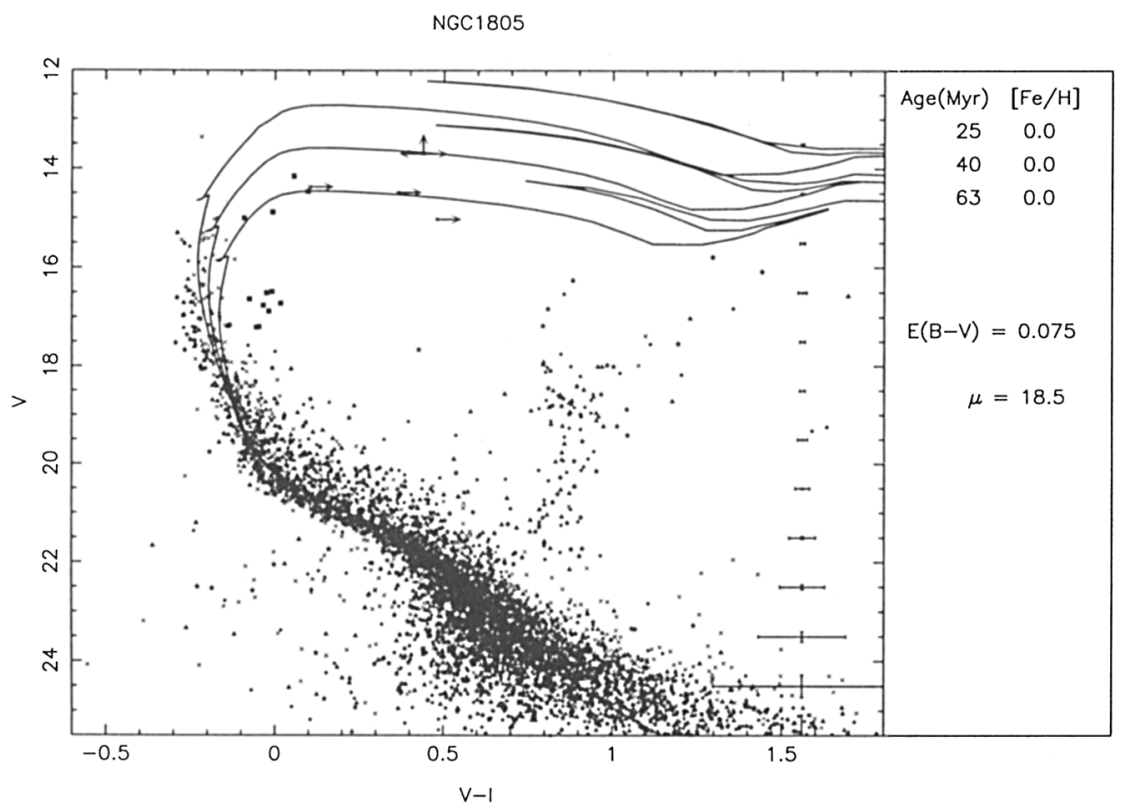

Figure 1. V vs. V-I CMD for NGC1805. Symbols denote data from individual WFPC2 chips. Bold squares mark Be stars. The isochrones shown are for ages 25,40 and 63 Myrs and metallicity $[\mathrm{Fe} / \mathrm{H}]=0$. (from Johnson et al. 2001 Fig. 3)

\section{Recent Results}

\subsection{Age Estimates for NGC1818 and NGC1831}

Observations of age spreads among the stars in a young cluster allow us to estimate the time scale and efficiency with which star formation took place (Elson, Fall \& Freeman 1987). In a young cluster the effect of an age spread on the CMD is to broaden the MS of the cluster. As Figure 1 illustrates, the problem of determining an age spread for the cluster stars is complicated by the presence of binary stars, blue stragglers and Be stars (shown as squares in Figure 1) all of which produce an apparent broadening around the MS turnoff which is not due to an inherent age spread (Johnson et al. 2001). While the Be stars can be identified by means of their $\mathrm{H} \alpha$ emission (Grebel, Richtler \& de Boer 1992), blue stragglers and binaries cannot be removed so easily.

Johnson et al. (2001) studied the CMDs of our two youngest clusters, NGC1818 and NGC1831, in order to determine their ages. By means of detailed population synthesis work they found that the clusters were best fit by a 50 per cent mixture of stars of ages 25 and 40 Myrs suggesting a 15 Myr age spread among the cluster stars. As this spread is $\sim$ a few dynamical times for these clusters, Johnson et al. conclude that it is not possible to discriminate unambiguously between efficient and inefficient star formation in these clusters, as both modes of formation could produce a bound cluster in this case. 


\subsection{The Binary Fraction of NGC1818}

The young cluster NGC1818 displays a clear binary sequence parallel to its MS but vertically offset by $\sim 0.75 \mathrm{mag}$. Elson et al. (1998) showed that this sequence implies a population of binaries with mass ratios $>0.7$, and a binary fraction which ranges from $20 \pm 5$ per cent in the outer parts to $35 \pm 5$ per cent in the inner parts. An analysis of the radial variation in the binary fraction of NGC1818 indicates that the brighter (more massive) objects are more centrally concentrated than the fainter objects, providing direct evidence for mass segregation in the cluster. An important question is whether or not this mass segregation is primordial - Elson et al. used $N$-body simulations to demonstrate that despite the young age of the cluster, dynamical effects could have produced such segregation from an initially radius independent distribution.

\subsection{Deep Luminosity Functions}

Santiago et al. (2001) obtained deep LFs for six of the clusters in our sample and found that within each cluster the LFs show a systematic increase in slope with increasing radius. This trend indicates a reduction in the relative number of high mass stars in the outer parts of the clusters and may be indicative of dynamical mass segregation or of preferential ejection of low mass stars from the inner regions.

\section{N-body Simulations}

$N$-body simulations of star clusters are entering a very exciting era, as recent advances in both computer hardware and software mean that we will soon be able to perform realistic simulations of clusters containing $\sim 50000$ stars. The simulations described below were for clusters of 5000 stars and were performed using Aarseth's NBODY4 code (Aarseth 1999), a direct $N$-body code written for the HARP special purpose computer (Makino, Kokubo \& Taiji 1993). As well as including all features of the dynamical evolution of star clusters, NBODY4 incorporates the stellar and binary evolution algorithms of Hurley, Pols \& Tout (2000). We have recently modified the code to include time-dependent external tidal fields.

In each simulation, the model cluster is initially in virial equilibrium and has an isotropic velocity distribution and a density which follows a Plummer law. The IMF of Kroupa, Tout \& Gilmore (1993) is used to generate the initial stellar masses, and a metallicity of $Z=0.02$ is assumed. Except for the clusters on elliptical orbits, a linearised approximation to the LMC tidal field is used (see e.g. Elson, Fall \& Freeman 1987).

In the following section we focus on our recent attempts to understand the origin of the core radius/age relation observed for LMC clusters. Observationally, the core radius is defined to be the radius at which the luminosity of the cluster has fallen to half its central value. In a simulated cluster containing only 5000 stars, the luminosity of the central parts may be dominated by one or two bright stars, making this observational definition difficult to apply in a robust manner. Instead, we use the definition of the density radius taken from Casertano \& Hut (1985) which for a Plummer model gives a value close to that 


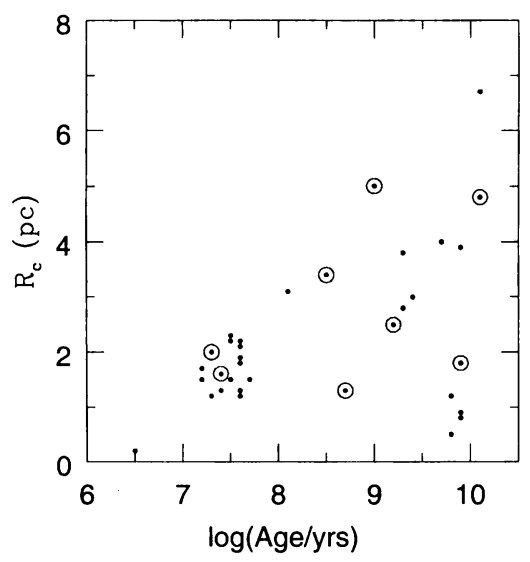

Figure 2. Observed core radii of rich LMC clusters plotted as function of age. Circled data points represent the clusters in our sample. Data taken from Elson (1991).

of the observational core radius. We also note that, due to the limited numbers of stars in the simulations completed to date, our simulations do not reproduce core radii as large as those in the observational sample.

\section{The Core Radius versus Age Relation}

Figure 2 shows the core radii for a sample of rich LMC clusters plotted as a function of their ages. The younger clusters all have similar, small core radii, while the older clusters exhibit a spread in core radius which increases with cluster age. In order to emphasise the puzzling nature of this trend we consider the clusters NGC1831 and NGC1868, the pair of circled clusters in the figure at an age of around $10^{8.5}$ years. These clusters have comparable total masses and metallicities and are at roughly the same radial distance from the LMC. It is therefore far from clear why these clusters should have core radii which differ by almost a factor of 3 .

As discussed by Elson (1991), intercluster IMF variations could produce the observed trend in core radius. A cluster with a flatter IMF than the standard Salpeter slope will contain a higher fraction of massive stars whose evolution has a significant impact on the evolution of the cluster core as supernova explosions and mass loss from these stars cause the core to grow in size. However, as there is now growing evidence pointing towards the universality of the IMF (see e.g. Gilmore 2001), it is worthwhile to look for other possible explanations for the observed trend. In particular, we have been studying the effects of cluster dynamics on the observed core radii.

While in young clusters it is possible to observe directly a binary sequence and hence to determine the binary fraction (see above), in older clusters such as NGC1831 and NGC1868 the MS is almost vertical and so unresolved binaries are difficult to detect. Thus, binary fraction variations might be involved in 

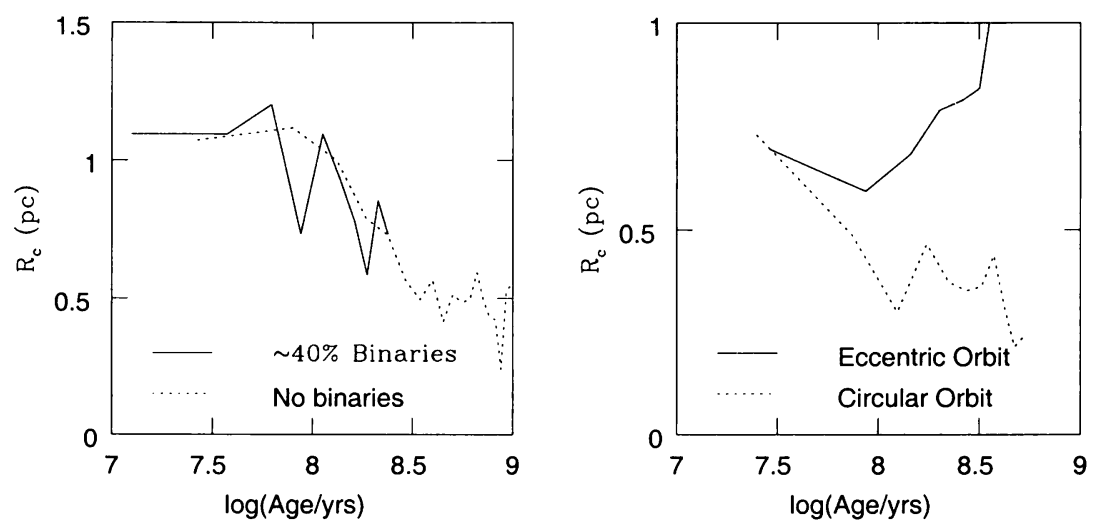

Figure 3. Core radii versus age for $N$-body simulated clusters containing 5000 stars each. Left: varying binary fractions, assuming a constant, linearised LMC tidal field. Right: clusters on circular and elliptical orbits about a point mass LMC. Each plotted curve represents the averaged results of several runs.

producing the observed trends in core radius, given the important role played by binaries in core evolution (see e.g. Meylan \& Heggie 1997). The left panel of Figure 3 shows the core radii of simulated clusters containing 5000 stars. The dashed line is for the case in which all the stars are single stars while the solid line shows the effect of putting $40 \%$ of the stars into binaries. As the figure shows, the binary fraction of the cluster does not appear to play a dominant role in the early ( $t<$ relaxation time) evolution of the cluster core radius.

The other main uncertainty in the observed cluster sample is the nature of the orbits on which the clusters move about the LMC. Clusters on circular orbits will experience a constant tidal field due to the LMC while clusters on more elongated orbits will experience a time varying field. As a first attempt to study this problem, we model the LMC as a point mass and study the evolution of clusters on elliptical orbits about this point mass. The right panel of Figure 3 compares the evolution of the core radii of clusters on circular orbits with those on slightly elliptical orbits. The elliptical orbits in this case have peri-LMC distances of $4 \mathrm{kpc}$ and apo-LMC distances of $8 \mathrm{kpc}$. The evolution of the core radius is significantly different in the two cases suggesting that differences in the orbits of LMC clusters might account for the observed core radius trend. The larger spread in core radii observed among older clusters would then imply a greater spread in orbital eccentricities for these clusters.

The orbits of the LMC clusters are usually assumed to be roughly circular (e.g. Elson, Fall \& Freeman 1987). In this context, however, it is important to note that the LMC and SMC form a binary pair of galaxies, and they undergo close encounters on a time scale of a few hundred Myrs (Zhao 1998). During the last of these encounters the distance of closest approach was only $\sim 6 \mathrm{kpc}$. Bearing in mind that the LMC is approximately 10 times more massive than the SMC, the effect of the SMC on the orbits of LMC clusters will be perturbative rather than disruptive. Nonetheless, during a close encounter, the SMC could 
easily move some of the LMC clusters onto eccentric orbits. We emphasize that the assumption of elliptical orbits for the clusters is meant only as an illustration of the effects of a time varying tidal field on the evolution of the cluster core radius, such as might be expected for a cluster which initially formed on a circular orbit and moved onto an orbit which brings it closer to the LMC, following a LMC-SMC close encounter.

\section{Future work}

The next generation of special purpose computers, GRAPE 6, will be available later this year. Using this machine we will be able to perform realistic simulations of clusters containing $\sim 50000$ stars allowing us to verify the results presented in Section 4 (Wilkinson, Hurley \& Gilmore 2001). A more realistic treatment of the LMC tidal field will also be included in future runs and the true magnitude of the perturbative effect of LMC-SMC close encounters on the orbits of LMC clusters will be investigated. Other work in progress includes a detailed study of mass segregation in our clusters, and an attempt to address the issue of converting LFs to mass functions (de Grijs et al. 2001).

\section{References}

Aarseth, S. 1999, PASP, 111, 1333

Beaulieu, S. F., Elson, R. A. W., Gilmore, G. F., Johnson, R. A., Tanvir, N., \& Santiago, B. 1999, in IAU Symp. 190, New Views of the Magellanic Clouds, eds. Y.-H. Chu, N. B. Suntzeff, J. E. Hesser \& D. A. Bohlender (SanFrancisco: ASP), 460

Casertano, S., \& Hut, P. 1985, ApJ, 298, 80

de Grijs, R., et al. 2001, in preparation

Elson, R. A. W. 1991, ApJS, 76, 185

Elson, R. A. W., Fall, S. M., \& Freeman, K. C. 1987, ApJ, 323, 54

Elson, R. A. W., Sigurdsson, S., Davies, M., Hurley, J., \& Gilmore, G. 1998, MNRAS, 300, 857

Gilmore, G. F. 2001, astro-ph/0102189

Grebel, E., Richtler, T., \& de Boer, K. S. 1992, A\&A, 311, 470

Hurley, J. R., Pols, O. R., \& Tout, C. A. 2000, MNRAS, 315, 543

Johnson, R. A., Beaulieu, S. F., Gilmore, G. F., Hurley, J., Santiago, B. X., Tanvir, N. R., \& Elson, R. A. W. 2001, MNRAS, 324, 367

Kroupa, P., Tout, C. A., \& Gilmore, G. F. 1993,MNRAS, 262, 545

Makino, J., Kokubo, E., \& Taiji, M. 1993, PASJ, 45, 349

Meylan, G., Heggie, D. 1997, A\&ARv, 8, 1

Santiago, B., Beaulieu, S. F., Johnson, R. A., \& Gilmore, G. F. 2001, A\&A, 369, 74

Wilkinson, M. I., Hurley, J. \& Gilmore, G. F. 2001, in preparation

Zhao, H. 1998, ApJ, 500, L149 


\section{Discussion}

J. Frogel: You used isochrones with solar $[\mathrm{Fe} / \mathrm{H}]$ to fit the cluster CMDs. From $\mathrm{H}$ II regions, the current $[\mathrm{Fe} / \mathrm{H}]$ in the LMC is more like $1 / 2$ solar. What effect would this have on what you showed?

M. Wilkinson: This is an interesting point. Using the Bertelli isochrones, the solar metallicity isochrones fit better than lower metallicity ones. However, it would be useful to repeat this comparison using other sets of isochrones, to check the robustness of our conclusions.

J. Maiz-Apellaniz: On what timescale would a massive halo (like that in 30 Dor around R136) be disrupted by tidal forces?

M. Wilkinson: Loosely bound (or marginally unbound) halos of stars can survive for a relatively long time - certainly they can remain near the cluster for several cluster orbital timescales.

B. Bohannan: This relates to Henny Lamer's question to Da Costa about star formation in the field in the LMC age gap - the clusters may have dissipated. A significant fraction of the present population of O-type stars in the LMC are not found in clusters or associations, yet they had to be formed in clusters or associations. Any thoughts on how the dispersion of these massive stars came to be?

M. Wilkinson: I think that the different timescales mean that O-type stars (with lifetimes < 10 Myrs) are unlikely to be associated with the LMC-SMC interaction (last close encounter $\sim 200 \mathrm{Myr}$ ago) or with disrupted clusters from the age gap (the age gap is at $\sim 10^{9.5} \mathrm{yr}$ ).

J. Brodie: In your age vs. cluster size plot, what is the distribution of metallicity at a given age? You mentioned that the central pair has similar metallicities but I am interested in the metallicity spread at the age extremes.

M. Wilkinson: Both our young clusters (NGC 1805, NGC 1818) have metallicities of order -0.4 to -0.7 . The oldest clusters both have metallicities of order -2.0 . Thus, among our clusters there is no significant metallicity spread (given the large uncertainties in the metallicity estimates) at either of the extremes in age, which is what motivated us to look for other explanations for the core radius trend. 